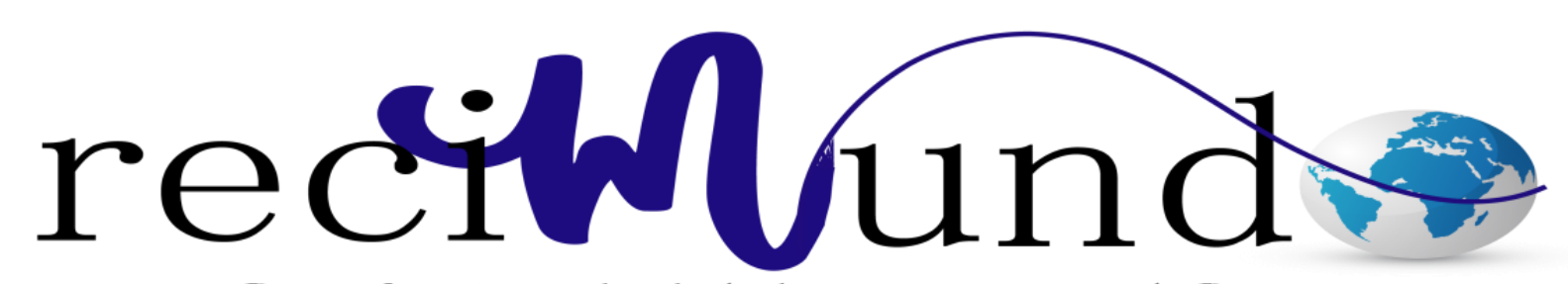

Revista Científica Mundo de la Investigación y el Conocimiento

Génesis Carolina Pérez Cruz a ${ }^{\text {; }}$ Elizabeth Stefania Molina Vega ${ }^{\text {b; }}$ Raquel Alexandra Colcha González ${ }^{\mathrm{c}}$

Astrocitoma fibrilar difuso

Diffuse fibrillary astrocytoma

Revista Científica Mundo de la Investigación y el Conocimiento. Vol. 3 núm.3. Esp., noviembre, ISSN: 2588-073X, 2019, pp. 853-875

DOI: $10.26820 /$ recimundo/3.(3.Esp).noviembre.2019.853-875

URL: http://recimundo.com/index.php/es/article/view/629

Código UNESCO: 3205 Medicina Interna

Tipo de Investigación: Artículo de Revisión

(C) RECIMUNDO; Editorial Saberes del Conocimiento, 2019

Recibido: 15/09/2019

Aceptado: 23/10/2019

Publicado: 30/11/2019

Correspondencia: genesisperez0926@gmail.com

a. Médico; Investigadora Independiente; Guayaquil, Ecuador; genesisperez0926@ gmail.com

b. Médico; Investigadora Independiente; Guayaquil, Ecuador; elizabeththmv.md@ gmail.com

c. Médica; Investigadora Independiente; Guayaquil, Ecuador; rcolchaczs5@gmail.com 


\section{Astrocitoma fibrilar difuso}

Vol. 3, núm. 3 Esp., (2019)

Génesis Carolina Pérez Cruz; Elizabeth Stefania Molina Vega; Raquel Alexandra Colcha González

\section{RESUMEN}

El objetivo del presente artículo es analizar el astrocitoma fibrilar difuso, su metodología corresponde al tipo documental, apoyado en fuentes bibliográficas a través de lecturas y revisión desde un manejo de textos, material impreso y digital, técnicas que ayudan a plasmar las respectivas consideraciones generales vinculadas con el tema previamente seleccionado. Consideraciones que permiten resaltar que el cáncer de cerebro primario se origina en el parénquima del cerebro, la médula espinal o nervios. También se encuentran algunos tumores que surgen a partir de las envolturas del cerebro o la médula (meninges) se consideran tumores cerebrales primarios. El riesgo global durante la vida de padecer un tumor cerebral es inferior al 1\%. Hay diferentes tumores cerebrales, según las células que resultan afectadas; entre los cuales se precisan los astrocitoma fibrilar difuso, que constituyen los gliomas de bajo grado más frecuentes y afectan a niños y adultos jóvenes entre 20 y 40 años. Debido a su naturaleza difusa presenta cierta tendencia a infiltrar el tejido cerebral circundante, también evidencian localización hemisférica en sustancia blanca, pero pueden aparecer como lesiones focales circunscritas; que pueden afectar al córtex adyacente, y tienen especial predilección por áreas funcionales secundarias como el área motora y el lóbulo de la ínsula, razón por la cual, resulta difícil de extirparlo todo, aunque su desarrollo es lento y puede pasar años antes de que la enfermedad vuelva a aparecer; el plantearse una nueva intervención resulta una práctica quirúrgica limitada, pues, existen secuelas neurológicas que no se consideran justificable, por ello, se recurre al uso de la radioterapia y quimioterapia con el fin de ofrecer años adicionales de vida relativamente libre de síntomas; es decir, reducir las crisis epilépticas.

Palabras Claves: Astrocitoma; Fibrilar; Difuso; Cáncer; Cerebral. 


\begin{abstract}
The objective of this article is to analyze the diffuse fibrillate astrocytoma, its methodology corresponds to the documentary type, supported by bibliographic sources through readings and review from a management of texts, printed and digital material, techniques that help the respective general considerations related to the subject previously selected. In the parenchyma of the brain, spinal cord or nerves. Some tumors that arise from brain or marrow (meninges) are also considered primary brain tumors. The overall lifetime risk of developing a brain tumor is less than $1 \%$. There are different brain tumors, depending on the cells that are affected; which requires diffuse fibrillate astrocytoma, which is the most common low-grade gliomas and affects children and young adults between the ages of 20 and 40. Due to its diffuse nature presents some tendency to infiltrate surrounding brain tissue, they also show hemispheric location in white matter, but may appear as circumscribed focal lesions; that can affect the adjacent cortex, and have special predilection for secondary functional areas such as the motor area and insule lobe, which is why, it is difficult to remove everything, although its development is slow and it may be years before the disease reappears; the consideration of a new intervention is a limited surgical practice, because, there are neurological squeal that are not considered justifiable, therefore, the use of radiation therapy and chemotherapy is used for the purpose.
\end{abstract}

Key Words: Astrocytoma; Fibrillar; Diffuse; Brain cancer. 


\section{Astrocitoma fibrilar difuso}

Vol. 3, núm. 3 Esp., (2019)

Génesis Carolina Pérez Cruz; Elizabeth Stefania Molina Vega; Raquel Alexandra Colcha González

\section{Introducción.}

En el campo de la medicina y especialmente en el área de oncología, al hacer referencia a los astrocitoma, se identifican como toda aquella neoplasia o tumor formada principalmente por astrocitos, uno de los principales tipos de células gliales que alimentan y dan soporte a las neuronas. Dicho tumor es una masa de astrocitos que se produce ante un crecimiento y proliferación anómala, patológica y descontrolada de uno de los tipos de tejido glial presentes en el sistema nervioso, tratándose de uno de los principales tipos de tumor cerebral.

Los astrocitomas son tumores cuyos síntomas pueden variar enormemente en función de la localización o localizaciones en las que aparezcan, si comprimen otras áreas cerebrales, su capacidad proliferativa o si se infiltran en otros tejidos o se autolimitan. Sin embargo, a nivel general es habitual encontrar la existencia de cefaleas, náuseas y vómitos, sonolencia, fatiga, alteración de la conciencia y confusión. También es relativamente frecuente que aparezcan alteraciones de la personalidad, conducta, fiebre y mareos son también habituales, así como alteraciones de peso sin razones para ello, problemas de percepción, lenguaje, pérdidas de sensibilidad y movilidad, e incluso convulsiones. De igual manera, al presentarse en niños, se generen malformaciones que alteran la anatomía cráneo facial tanto intracraneal como extra craneal.

Este tipo de tumoración puede presentarse, al igual que ocurre con otros tumores, de manera circunscrita a una región o estructura concreta o difusa, teniendo el primero mejor pronóstico que la segunda. Por lo general no suelen producir metástasis hacia otras áreas del organismo más allá del sistema nervioso, si bien sí se expanden dentro de dicho sistema. Su 


\section{Astrocitoma fibrilar difuso}

Vol. 3, núm. 3 Esp., (2019)

Génesis Carolina Pérez Cruz; Elizabeth Stefania Molina Vega; Raquel Alexandra Colcha González

diagnóstico es complejo, siendo necesario realizar diversos procedimientos. En primer lugar, efectuar una biopsia del tumor, la cual permitirá seleccionar una muestra del tumor cerebral, en la actualidad es realizada mediante estereotaxia. Posteriormente se cumple con un análisis fenotípico e histológico para comprobar el tipo de tejido y comportamiento. Finalmente hay que valorar la capacidad proliferativa, con el fin de poder determinar su grado y capacidad de infiltración.

Al igual que ocurre con el resto de tumores cerebrales, las causas de los astrocitomas son en su mayoría desconocidas. Sin embargo, algunos trastornos hereditarios pueden facilitar su aparición, como por ejemplo la neurofibromatosis. También la infección con el virus EpsteinBarr o la aplicación previa de radioterapia debido a otro tumor parecen ser factores de riesgo para desarrollarlos. De acuerdo con la clasificación expuesta por la Organización Mundial de la Salud existen cuatro grados; siendo de interés para el presente artículo el grado II, en este grupo se incluyen los astrocitomas difusos, entre los más habituales se encuentran: los astrocitomas fibrilar, gemistocítico y protoplasmático. Son el tipo de astrocitoma de bajo grado más habitual.

Según Louis (2016) los astrocitomas difusos "afectan sobre todo a niños y adultos jóvenes entre 20 y 40 años, aunque en estudios recientes se ha observado un aumento progresivo de su incidencia en pacientes mayores de 60 años". (p.16). Es decir, esos tumores se observan fundamentalmente en los hemisferios cerebrales. Son tumores sólidos, pero pueden tener degeneración micro quística. Habitualmente son hipercelulares y presentan atipia nuclear. Infiltran la sustancia blanca y pueden producir satelitosis cuando los astrocitos rodean a las neuronas. 


\section{Astrocitoma fibrilar difuso}

Vol. 3, núm. 3 Esp., (2019)

Génesis Carolina Pérez Cruz; Elizabeth Stefania Molina Vega; Raquel Alexandra Colcha González

Es importante destacar que, debido a su carácter difuso, el crecimiento lento, el miedo a las secuelas de una cirugía más agresiva y el dudoso papel de la radioterapia en la supervivencia global del paciente, su manejo es controvertido. Entre los factores pronósticos se encuentran el grado de resección quirúrgica, patrones moleculares favorables (IDH1/2 positivo, presencia de la mutación 1p19q) o desfavorables (histología de astrocitoma, o predominio gemistocítico), localización en áreas elocuentes, edad avanzada, tamaño, extensión a través de la línea media, presencia de déficit neurológico al diagnóstico, ausencia de captación de contraste o ausencia de perfusión tumoral en secuencias RM.

Por lo tanto, la atención médica se convierte en un factor esencial, dado que, a través de diagnóstico se puede llegar a tener un manejo de pacientes con astrocitoma fibrilar difuso, este proceso es un tema complejo que debe ser cuidadosamente individualizada. De allí, que la terapia multimodal que comprenden la resección quirúrgica, radioterapia, y quimioterapia juegan un papel importante; los beneficios de cada uno deben ser equilibrados cuidadosamente contra los riesgos o complicaciones asociadas con su uso. El objetivo inicial es controlar los síntomas, reducir el volumen del tumor, y obtener un diagnóstico histológico y genético claro. La cirugía permanece como una herramienta importante de diagnóstico y modalidad terapéutica. Su objetivo es proveer tejido adecuado para el diagnóstico histopatológico y adecuada gradificación. Las biopsias con aguja son realizadas cuando las lesiones son profundas o se encuentran en regiones críticas del cerebro.

Es importante acotar que, estos tumores cerebrales tienen elevada tendencia a reproducirse una vez finalizado el tratamiento o incluso durante el mismo. La recaída de un tumor cerebral puede ocurrir entre meses y años después según el tipo de tumor. En caso de 


\section{Astrocitoma fibrilar difuso}

Vol. 3, núm. 3 Esp., (2019)

Génesis Carolina Pérez Cruz; Elizabeth Stefania Molina Vega; Raquel Alexandra Colcha

González

recaída, el paciente deberá ser evaluado por un equipo multidisciplinar, es decir por los diferentes especialistas implicados en el diagnóstico y tratamiento de los tumores cerebrales. Se plantearán las diferentes opciones que pueden ser en ocasiones quirúrgicas de nuevo y/o radioterápicas y/o sistémicas en función de cada caso y de cada tumor.

Según los eventos planteados, se puede resaltar que, al contrario que los astrocitomas de grado I los de grado II sí son propiamente cáncer, siendo tumores malignos. Los astrocitomas de grado dos agresivos, mucho más complejos de tratar y considerándose malignos y expansivos. Suelen generar crisis epilépticas, observándose lesiones difusas. Generalmente en este grupo se incluyen los astrocitomas difusos, entre los cuales los más habituales podemos encontrar los astrocitomas fibrilar, gemistocítico y protoplasmático. Son el tipo de astrocitoma de bajo grado más habitual. En relación al tratamiento temprano con frecuencia mejora la posibilidad de un buen desenlace clínico. El tratamiento depende del tamaño, tipo de tumor y de su salud general. Los objetivos del tratamiento pueden ser curar el tumor, aliviar los síntomas y mejorar la actividad cerebral o el bienestar. Con frecuencia, se requiere la cirugía para la mayoría de los tumores cerebrales primarios. Algunos se pueden extirpar totalmente. En aquellos casos, cuando los tumores están muy profundos dentro del cerebro o se han infiltrado en el tejido cerebral, se puede llevar a cabo la citorreducción quirúrgica, en lugar de extirparlos. La citorreducción quirúrgica es un procedimiento para reducir el tamaño del tumor.

Estas consideraciones, resultan un aporte significativo para el desarrollo temático propuesto, mediante ello, se logra visualizar nuevas interpretaciones de interés en el campo de la medicina y convertirse en un aporte para la realización de nuevas indagaciones que representen importancia en el estudio de los tumores cerebrales. 


\section{Astrocitoma fibrilar difuso}

Vol. 3, núm. 3 Esp., (2019)

Génesis Carolina Pérez Cruz; Elizabeth Stefania Molina Vega; Raquel Alexandra Colcha González

\section{Metodología.}

La introducción del conocimiento científico es un proceso inacabado, por lo tanto, es objeto de revisión y producción constante, las utilidades de los métodos en el campo de la metodología tienen que revisarse de manera regular, con el propósito de validar su eficacia. En consecuencia, los desafíos de la investigación científica exigen que tanto los métodos como la metodología sean entendidos y asumidos como herramientas útiles que permiten el abordaje, comprensión y argumentación de los problemas de estudio.

En consecuencia, el conocimiento científico se caracteriza por exigir el estudio, análisis y explicación de los hechos que se definen como problemas. Es un tipo de conocimiento que no admite aprioris ni valoraciones precipitadas, sino que se deben utilizar conceptos, metodologías y teorías para trabajar con la realidad entendida como problema. Lo importante es que su producción es fruto de indagaciones y descubrimientos que llaman la atención de quien investiga. Combina curiosidad sistematizada, así como preguntas específicas para identificar el sentido de los fenómenos, en términos de su causalidad, consecuencias e impacto. Al respecto, Aguilera (2018) concibe al método como "aquel que permite simplificar la complejidad al seleccionar los elementos más significativos de un problema a fin de proceder a su estructuración conceptual y explicación casual”. (p.59).

En este caso particular, se considera pertinente la utilización del método comparativo, mediante su utilización el investigador tiene oportunidad de desglosar los aspectos conceptuales, para luego explicar las interrelaciones causales que se registran en su contenido, de este modo, hace posible en dotar de propiedades cognoscitivas que permiten el abordaje ordenado de una 


\section{Astrocitoma fibrilar difuso}

Vol. 3, núm. 3 Esp., (2019)

Génesis Carolina Pérez Cruz; Elizabeth Stefania Molina Vega; Raquel Alexandra Colcha González

parte de la realidad y que depende del sujeto cognoscente la utilidad que pueda tener al conseguir a través del trabajo de investigación, es posible esclarecer lo que antes no se conocía. Es así como Aguilera (ob.cit) define al método comparativo "como una actividad particular que ayuda al investigador a establecer de forma apropiada las diferencias y semejanzas presente en un tema en estudio". (p. 61).

Tipo de Investigación

Para lograr la producción del conocimiento científico, se hace necesario establecer una dirección metodológica en correspondencia con los objetivos del estudio, por lo tanto, en este caso particular, se vincula con la investigación documental, apoyada en generar desde la revisión bibliográfica nuevas consideraciones generales para construir un tema que tenga importancia en el campo de la medicina, pues, al analizar la astrocitoma fibrilar difuso. Es así como, Aguilera (ob.cit) precisa que la investigación documental "es una estrategia en la que se observa, reflexiona sistemáticamente sobre realidades teóricas o empíricas, usando diferentes documentos donde indaga, interpreta, presenta datos e información sobre un tema determinado de cualquier ciencia": (p. 64).

De lo antes planteado, se precisa que, al adecuar la indagación mediante la utilización de la investigación documental, lleva a considerar su importancia en el campo científico, al lograr un nuevo conocimiento relacionado con la incidencia de eventos adversos relacionados con el análisis referido al tema seleccionado. 


\section{Astrocitoma fibrilar difuso}

Vol. 3, núm. 3 Esp., (2019)

Génesis Carolina Pérez Cruz; Elizabeth Stefania Molina Vega; Raquel Alexandra Colcha González

\section{Técnicas Documentales}

La formación de conceptos normativos y aplicados que se enlazan con la sociedad, por su complejidad, demanda no sólo la multiplicación del saber científico, sino que el mismo debe aplicarse para solucionar los problemas de la vida colectiva. Este hecho define el alcance del conocimiento científico para que no sea entendido de manera enciclopédica, sino que se orienta a generar utilidad aplicada. Por lo tanto, para darle flexibilidad al método es significativo, fijar una posición clara en cuanto a las técnicas documentales que sirven de apoyo para la redacción del artículo.

En razón de lo anterior, se puede indicar que mediante la incorporación de las técnicas documentales, se hace posible redactar los diferentes aspectos que constituyen el contenido del presente artículo, en consecuencia, debido al desarrollo tecnológico que existe actualmente permite el uso del internet como un lugar para tener acceso a textos originales, listas bibliográficas o sitios donde se puede buscar, consultar catálogos de grandes bibliotecas universitarias de todo el mundo e inclusive listados de títulos publicados por algunas editoriales, videos, fotografías, imágenes reales y hasta el periódico. Para Tejería (2018) las técnicas documentales "son los diferentes medios o recursos que le permiten al investigador, indagar en relación a un tema en especial.” (p.36).

\section{Técnicas de Recolección de Información}

Para el desarrollo del presente artículo, se hizo necesario nos sólo visualizar su ubicación en una metodología del conocimiento científico, sino agregar, el manejo efectivo de las técnicas de recolección de información, para ello, se incorporó la lectura reflexiva como parte 


\section{Astrocitoma fibrilar difuso}

Vol. 3, núm. 3 Esp., (2019)

Génesis Carolina Pérez Cruz; Elizabeth Stefania Molina Vega; Raquel Alexandra Colcha González

fundamental del trabajo monográfico. En esta dirección Tejería (ob.cit) las identifica como "un aporte interpretativo basado en la lectura como parte esencial de los elementos que estructuran un contenido en especial" (p.39). De este, modo, se analizaron de forma especial los aspectos que conforman al tema para responder a su objetivo.

\section{Resultados.}

Una vez planteado el objetivo del presente artículo, se precisan aquellos eventos relacionados con su contenido, mediante ello, se asegura el desarrollo de nuevos aspectos importantes para reportar la significación del tema.

\section{Astrocitoma Fibrilar Difuso}

El astrocitoma es un tipo de cáncer que se van formando a través de células llamadas astrocitos, normalmente en la médula espinal o en el cerebro. Es un tipo de glioma, un tumor glial, que suelen ser benignos y que se originan en las células de la estructura cerebral. Los astrocitomas son los más frecuentes y pueden aparecer a cualquier edad. No todos los astrocitomas son iguales. Tienen una velocidad de propagación muy diversa: pueden ser lentos o tremendamente agresivos. La velocidad de desarrollo es la que marca el tratamiento a seguir y pronóstico general.

Es importante destacar que, la clasificación realizada por la Organización Mundial de la Salud desde 1979, en cuanto a los tumores del sistema nervioso central, basado en la célula de origen del proceso tumoral y características morfológicas asociadas al pronóstico. Se estratifican entre tumores: 


\section{Astrocitoma fibrilar difuso}

Vol. 3, núm. 3 Esp., (2019)

Génesis Carolina Pérez Cruz; Elizabeth Stefania Molina Vega; Raquel Alexandra Colcha González

Grado I: Tumores circunscritos, lento crecimiento y bajo potencial de conversión a un tumor de mayor malignidad.

Grado II: Tumores de borde difuso, lento crecimiento y algunos, con tendencia a progresar a tumores de mayor malignidad.

Grado III: Tumores infiltrantes con células atípicas o anaplásicas y mayor número de mitosis.

Grado IV: tumores de rápido crecimiento con alta tasa mitótica, pudiendo presentar vasos de neo formación y áreas de necrosis.

La clasificación OMS presenta una implicancia terapéutica, pues las lesiones OMS grado I, es decir, circunscritas o de borde neto pueden ser removidas en su totalidad a través de la neurocirugía; Las lesiones infiltrantes OMS II, III y IV, al presentar un borde difuso su exéresis suelen ser incompletas, por lo que tienen un seguimiento imagenológicas y específico en lesiones.

Al tomar en consideración la clasificación citada, se puede decir que no existe certeza sobre las causas principales de la aparición de este tipo de cáncer, aunque se ha determinado que algunos factores, como estar expuesto a radiaciones o tener antecedentes familiares, pueden aumentar las posibilidades de tener esta enfermedad. Entre los diferentes tipos se encuentran el Astrocitoma grado II o astrocitoma difuso. Existen diferentes subgrupos con características propias. Es un tumor infiltrante y suele evolucionar a una histología (estructura, composición y 


\section{Astrocitoma fibrilar difuso}

Vol. 3, núm. 3 Esp., (2019)

Génesis Carolina Pérez Cruz; Elizabeth Stefania Molina Vega; Raquel Alexandra Colcha

González

características de los tejidos orgánicos) agresiva. En este grupo se incluiría el xantoastrocitoma pleomórfico.

En tal sentido, García (2017), destaca que la presencia de los astrocitoma fibrilar difuso, se identifica como aquellos de bajo grado son menos comunes, son aproximadamente el $15 \%$ de los tumores del SNC diagnosticados en adultos cada año. El único factor de riesgo, es la exposición a radiación ionizante. Los factores hereditarios no juegan un papel importante, aunque estos tumores son más frecuentes en paciente con NF-1 y sd. Fraumeni (mutación que afecta al gen supresor tumoral TP53). La principal forma de presentación es en forma de crisis, que ocurren en el $80 \%$ de los casos, debido probablemente a la localización superficial y baja tasa de crecimiento. La mayoría permanecen asintomáticos incluso aunque tengan efecto masa, los síntomas de HTIC son poco frecuentes.

Este tipo de tumor ocurre en adultos, con pico de edad a finales de los 30 años, asientan frecuentemente en los hemisferios cerebrales (temporal, frontal posterior y lóbulo parietal anterior), la mayoría se presenta como crisis. Anaplasia y mitosis ausentes. Infiltran el tejido neural circundante. Mejor pronóstico si ocurre en jóvenes, peor pronóstico si HITC, alteraciones de la consciencia, cambios de personalidad, déficits neurológicos, captación de contraste. Según la histopatología, pueden dividirse en Fibrilar: surge de la profundidad de la sustancia blanca, es duro y correoso por fibrillas neurogliales que produce. Baja celularidad con mínima atipia nuclear. Es el subtipo más frecuente. 


\section{Astrocitoma fibrilar difuso}

Vol. 3, núm. 3 Esp., (2019)

Génesis Carolina Pérez Cruz; Elizabeth Stefania Molina Vega; Raquel Alexandra Colcha González

\section{Imagen 1. Astrocitoma Fibrilar Difuso}

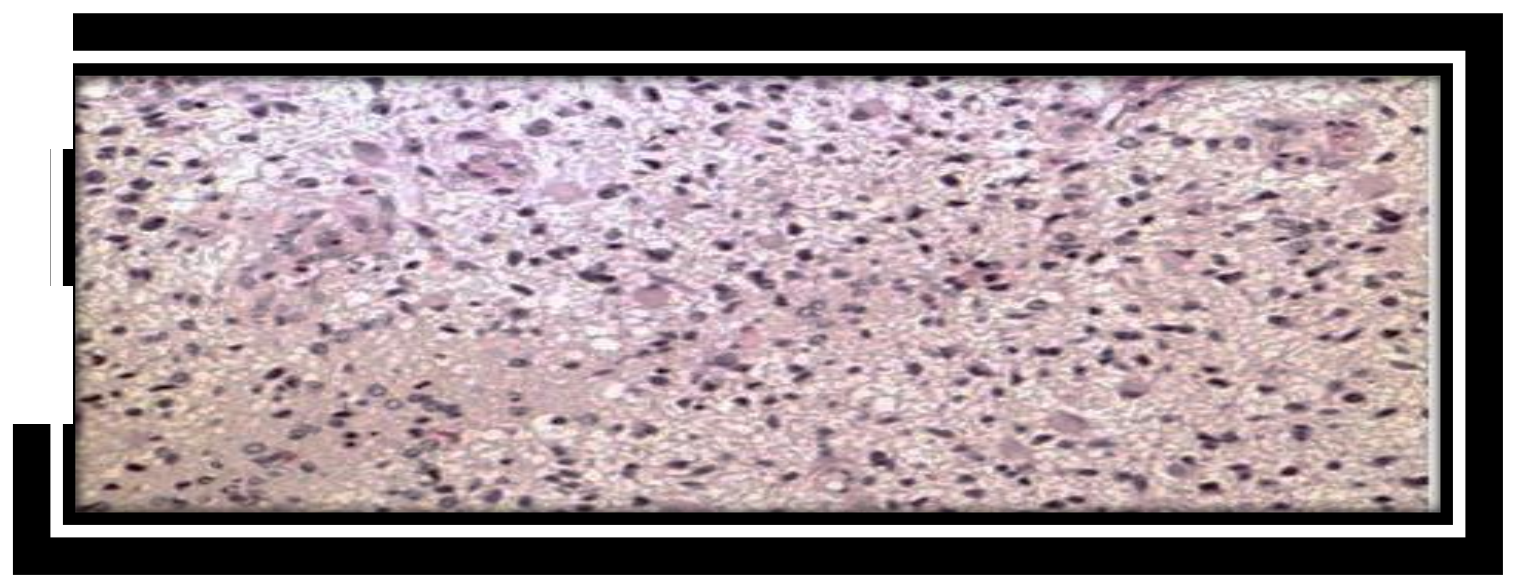

Fuente: García (ob.cit).

En cuanto a los síntomas que presenta el astrocitoma están en función de varios factores, pero especialmente de la localización del tumor. Si están en el cerebro suelen causar convulsiones, dolores de cabeza y vómitos. Los que están en la médula espinal pueden causar cierta discapacidad en la zona del cuerpo afectada por el tumor. Por ello, el médico ha de realizar como primer paso un examen neurológico en el que habla con el paciente y obtenga información acerca de su comportamiento y estado general. Después es imprescindible recurrir a las pruebas de diagnóstico por imágenes para confirmar el astrocitoma y revelar el estado real del tumor: tamaño, localización y velocidad de desarrollo del mismo.

Al no conocerse la causa exacta, no se puede establecer un protocolo de actuación eficaz. Es recomendable no exponerse de forma continuada a radiaciones porque está demostrado que son un factor de riesgo para el desarrollo de este tipo de cáncer. Sin embargo, hay una variedad de tratamientos en función del estado del paciente y de la velocidad de la enfermedad. La primera opción es la cirugía para intentar extraer todas las células cancerígenas o el máximo 


\section{Astrocitoma fibrilar difuso}

Vol. 3, núm. 3 Esp., (2019)

Génesis Carolina Pérez Cruz; Elizabeth Stefania Molina Vega; Raquel Alexandra Colcha González

número que sea posible. A veces no se puede acceder a células dañadas porque se encuentran en zonas muy delicadas del cerebro. La intervención quirúrgica es suficiente en algunos casos. Si quedan rastros del cáncer o no se ha podido realizar la intervención, entonces se recurre a la quimioterapia y radioterapia.

Los objetivos de la cirugía: confirmación histológica, mejoría neurológica del paciente, reducir el riesgo de crecimiento del tumor, prevenir la transformación maligna. La misma da un diagnóstico histológico y a su vez, puede ser terapéutica. En pacientes donde es posible una resección radical, la cirugía puede ser el único tratamiento. La resección total a menudo no es posible llevarla a cabo sin secuela neurológicas por la naturaleza infiltrativa del tumor. En estas situaciones está indicada RT y QT neoadyuvante. En pacientes con buen pronóstico (jóvenes, solo presentan crisis) la observación tras la resección es una opción terapéutica. Palavero (2018) indica que entre el peor pronóstico: tumores $>4 \mathrm{~cm}$, astrocitomas u oligoastrocitomas peor que oligodendroglioma, enfermedad residual \&gt 1cm en RM. El uso de RT/QT en estos pacientes es incierto, pero normalmente se pospone hasta que haya progresión.

Según Angelis (2017), el síntoma de presentación más frecuente son las crisis epilépticas (65-95\%), aunque, se trata de tumores de crecimiento lento, con frecuencia los síntomas aparecen de forma insidiosa en forma de cefaleas (40\%), cambios de conducta (10\%), depresión, y pueden prolongarse varios años antes del diagnóstico. Asimismo, se presentan generalmente como lesiones difusas infiltrativas, pero pueden aparecer como lesiones focales circunscritas. Son tumores de localización hemisférica en sustancia blanca que pueden afectar al córtex adyacente, y que tienen especial predilección por áreas funcionales secundarias como el área suplementaria motora y el lóbulo de la ínsula. 


\section{Astrocitoma fibrilar difuso}

Vol. 3, núm. 3 Esp., (2019)

Génesis Carolina Pérez Cruz; Elizabeth Stefania Molina Vega; Raquel Alexandra Colcha González

De igual manera precisa el autor citado, que entre las características moleculares de los astrocitoma fibrilar difuso la anomalía genética más común es la alteración de TP53 (> 59\%). Como resultado de la interrupción de la cascada de las vías de transducción de señales y proliferación celular, la división celular anormal facilita la inestabilidad genómica y la transformación anaplásica. Es importante resaltar también el reciente hallazgo de la presencia de mutaciones de la isocitrato dehidrogenasa 1 (IDH1) entre el 59 y el $90 \%$ de los tumores, mutación descrita como evento temprano en la tumorogénesis,

El astrocitoma difuso grado II es una lesión bien diferenciada y difusamente infiltrante que representa el 10-15\% de todos los tumores cerebrales astrocitarios. Afecta tanto a jóvenes como a adultos de edad media, encontrando un 60\% entre 20-45 años. Estos tumores se localizan de manera frecuente supratentorialmente, en lóbulos frontal y temporal (3). Histológicamente, el astrocitoma difuso de bajo grado está formado por lesiones con aumento de celularidad, núcleos ovales, escasa atipia y mitosis ausentes. Pueden ser difícilmente diferenciables de astrocitos normales o reactivos.

De igual manera complementa el autor que, los tumores astrocíticos representan aproximadamente el $40 \%$ de todas las neoplasias del Sistema Nervioso Central (SNC) dela edad pediátrica. Aproximadamente 15 a $25 \%$ son astrocitomas de bajo grado y 10 a15\% de alto grado, $10-20 \%$ son astrocitomas del talo cerebraly 10-20\% astrocitomas cerebrales. Los factores de riesgo aumentan la probabilidad de que una persona desarrolle un tumor. Si bien los factores de riesgo a menudo influyen en el desarrollo de un tumor, la mayoría no es una causa directa de su formación. Algunas personas que tienen varios factores de riesgo nunca desarrollan un tumor, mientras que sí lo hacen otras personas sin factores de riesgo conocidos. Los médicos e 


\section{Astrocitoma fibrilar difuso}

Vol. 3, núm. 3 Esp., (2019)

Génesis Carolina Pérez Cruz; Elizabeth Stefania Molina Vega; Raquel Alexandra Colcha

González

investigadores aún no conocen las causas de la mayoría de los tumores cerebrales. Existe evidencia de que la genética puede intervenir en un pequeño porcentaje.

En este mismo orden de ideas, se puede decir que las opciones terapéuticas en los astrocitoma fibrilar difuso son: observación, cirugía, radioterapia y quimioterapia. La observación estricta, con controles radiológicos periódicos, se recomienda en pacientes asintomáticos o mínimamente sintomáticos, e incluso en aquellos con crisis epilépticas al diagnóstico, pero bien controladas con tratamiento médico. La observación está contraindicada en aquellos pacientes cuya sintomatología no se controla con tratamiento médico. Por ello, la cirugía permite la obtención de histología para llegar al diagnóstico definitivo y caracterización molecular del tumor, y es la primera opción terapéutica en la mayoría de los casos. Sin embargo, aun en el caso de conseguirse una resección macroscópicamente completa, debe mantenerse un estrecho control, dado que, en pacientes <40 años intervenidos de astrocitomas de bajo grado no pilocíticos, se ha evidenciado una tasa de progresión tumoral de más del $50 \%$ a los 5 años. El momento óptimo para iniciar la radioterapia y las dosis más adecuadas para el tratamiento aún son objeto de discusión. En general, la radioterapia puede diferirse en pacientes en buena situación clínica, siempre que se haga un seguimiento cuidadoso.

Los pacientes mayores de 40 años, los pacientes con tumores grandes irresecables y los que presentan déficit neurológico, son habitualmente tratados con radioterapia precoz. En cuanto al uso de quimioterapia, aunque no se dispone de evidencia firme para su uso adyuvante postquirúrgico (se ha observado beneficio en la supervivencia libre de progresión, pero no en la supervivencia global), hay ensayos clínicos prospectivos en marcha para evaluar esta indicación. 


\section{Astrocitoma fibrilar difuso}

Vol. 3, núm. 3 Esp., (2019)

Génesis Carolina Pérez Cruz; Elizabeth Stefania Molina Vega; Raquel Alexandra Colcha González

En el momento de la progresión puede estar indicado el tratamiento quimioterápico o la reirradiación

\section{Cáncer de Cerebro}

A nivel mundial el cáncer es una de las principales causas de mortalidad, responsable de 8.2 millones de muertes el año 2012. Se determina que el número de casos nuevos aumentará en un $70 \%$ en 20 años. En Chile el cáncer constituye la segunda causa de mortalidad con una tasa de 129.5 por 100000 habitantes el año 2007. Cabe destacar la baja tasa de verificación histológica llevado a cabo en las regiones de Antofagasta, los Ríos y la provincia del Bio-Bío, alcanza solo el $50 \%$ de los pacientes.

El cerebro es la estructura principal responsable de la coordinación y control del organismo. Se trata de una masa de tejido blanda protegida por los huesos del cráneo y unas membranas, las meninges. El líquido cefalorraquídeo fluye por el cerebro y discurre entre las meninges a través de otros espacios denominados ventrículos. No tiene drenaje linfático (salida a través de los ganglios) y diseminación a través de la sangre es muy rara, por lo que los tumores cerebrales primarios, es decir, los que se originan en el cerebro casi nunca se extienden al resto del organismo salvo en algún tipo muy concreto de tumor cerebral.

De igual manera, se puede indicar que es una estructura muy compleja que procesa y memoriza muchísima información de forma continuada. Está constituido por el encéfalo (hemisferios cerebrales, tronco del encéfalo y cerebelo y por la médula espinal que se encuentran alojados dentro de unas estructuras óseas que son el cráneo y la columna vertebral respectivamente. Está cubierto por las meninges, de las que existen tres capas (duramadre, 


\section{Astrocitoma fibrilar difuso}

Vol. 3, núm. 3 Esp., (2019)

Génesis Carolina Pérez Cruz; Elizabeth Stefania Molina Vega; Raquel Alexandra Colcha González

aracnoides y piamadre), que protegen al cerebro y a la médula espinal. El conjunto de todas estas diferentes partes se denomina Sistema Nervioso Central (SNC) encargado de controlar a través de diferentes capacidades mentales como la memoria, la inteligencia, el habla y las emociones y también sentidos como la visión el gusto, el tacto o el oído o también funciones básicas como la respiración o la frecuencia cardíaca.

Con la publicación del Genoma Humano y los continuos avances derivados de ello es posible que pueda encontrarse algún gen asociado al desarrollo de los tumores cerebrales los cambios genéticos en múltiples tumores entre ellos el glioblastoma (tipo de tumor cerebral) que pueden ayudar a explicar cómo se desarrollan. Cada célula del cuerpo humano tiene 23 pares de cromosomas y se ha visto que algunos tipos de tumores cerebrales pueden perder o ganar alguna pieza (brazo) de los mismos. Los cambios más conocidos y frecuentes descritos en algunos tumores cerebrales como los denominados oligodendrogliomas pueden ocurrir en los cromosomas 1 y 19 y los avances en el conocimiento de dichas alteraciones están ayudando en gran manera al tratamiento de los mismos.

De acuerdo con Barrientos (2017), los tumores primarios del Sistema Nervioso Central (SNC) constituyen alrededor del 3\% del total de las neoplasias del adulto y son responsables del 2,4\% de las muertes por cáncer. La tasa de incidencia de los tumores cerebrales es de 20 casos por cada 100.000 habitantes/año. La incidencia en niños (0-19 años) es de 5 casos, mientras en adultos (mayores de 20 años) es de 27 casos por cada 100.000 habitantes/año. En España se diagnostican 4.000 casos nuevos al año y se estima una incidencia de 8,73 en varones y de 5,41 en mujeres (1). En la infancia, se encuentra un pico de incidencia en los niños de entre 5 a 14 años, donde representan entre el 19-20\% de todos los cánceres; mientras que, en los adultos, la 


\section{Astrocitoma fibrilar difuso}

Vol. 3, núm. 3 Esp., (2019)

Génesis Carolina Pérez Cruz; Elizabeth Stefania Molina Vega; Raquel Alexandra Colcha González

mayor incidencia se sitúa por encima de los 50 años (edad media en los 56 años). De todas formas, la distribución por edad es variable y depende de la localización tumoral y la histología.

Asimismo, precisa que la localización de estos tumores es variable, aunque el sitio más común donde se encuentran es en las meninges (35\%). El 22\% de los tumores se localiza en el lóbulo frontal, temporal, parietal y occipital del cerebro mientras que los tumores situados en cerebro, ventrículo, cerebelo o tronco encefálico representan un 7,5\%. En los nervios craneales y la médula espinal se encuentra el $10 \%$ de todos los tumores ( $7 \%$ y $3 \%$, respectivamente). La pituitaria y la glándula pineal representan alrededor del 16\% de los tumores (1)

\section{Conclusiones.}

Los aspectos referidos a este apartado permiten indicar una serie de apreciaciones particulares como respuesta al objetivo que caracteriza al artículo, las mismas resulta una valoración interpretativa de los contenidos debidamente presentados. Entre ellas se encuentran las siguientes consideraciones:

El cerebro como estructura principal responsable de la coordinación y control del organismo. Se trata de una masa de tejido blanda protegida por los huesos del cráneo y unas membranas, las meninges. Sin embargo, al igual que los demás órganos del cuerpo humano, aparecen en su interior diferentes enfermedades, donde el cáncer se convierte en una de las más importantes. Por ello, la presencia de los tumores cerebrales amerita una serie de evaluaciones mediante técnicas innovadoras que hagan posible estimar su ubicación y así planificar el respectivo abordaje clínico. 


\section{Astrocitoma fibrilar difuso}

Vol. 3, núm. 3 Esp., (2019)

Génesis Carolina Pérez Cruz; Elizabeth Stefania Molina Vega; Raquel Alexandra Colcha González

Dentro de este orden de ideas, se encuentran los llamados astrocitoma biliar difuso, tumor cerebral que debido a su naturaleza de crecimiento lento, el paciente durante su evaluación presenta con frecuencia crisis epilépticas y la presencia de cefalea, su característica difusa se debe a estar en una localización infiltrativa hemisférica en sustancia blanca; sin embargo, también pueden tener aparición en lesiones focales circunscritas que afectan el córtex adyacente. Los mismos, se observan fundamentalmente en los hemisferios cerebrales. Son tumores sólidos, pero pueden tener degeneración microquística. Habitualmente son hipercelulares y presentan atipia nuclear. Infiltran la sustancia blanca y pueden producir satelitosis cuando los astrocitos rodean a las neuronas.

Ante estas características que lo identifican, las ciencias médicas asumen durante el diagnóstico la aplicación de técnicas innovadoras como resonancias, tomografía, biopsias entre otros, con el fin de estudiar las condiciones histológicas del tumor y estimar su ubicación, todo ello, hace posible programar la respectiva intervención como parte fundamental de tratamiento, la misma, va depender de las condiciones o profundidad y extensión que el tumor tengan, pues, es importante considerar las secuelas neurológicas como factores esenciales para mejorar la calidad de vida de paciente. También, existen terapias complementarias como: la radioterapia y quimioterapia.

Es importante destacar que estos astrocitomas pueden ser benignos, pero el caracterizado desde un punto médico fibrilar difuso en su mayoría lograr ocupar el grado II y puede asumir en el tiempo una tendencia hacia los grados subsiguientes (III y IV). De allí, que las nuevas investigaciones científicas en estos tumores cerebrales, fija posiciones claras en cuanto a la presencia de factores moleculares hereditarios, razón por la cual, hoy en día las ciencias 


\section{Astrocitoma fibrilar difuso}

Vol. 3, núm. 3 Esp., (2019)

Génesis Carolina Pérez Cruz; Elizabeth Stefania Molina Vega; Raquel Alexandra Colcha González

oncológicas y los cirujanos abren un nuevo proceso de diagnóstico donde se considera el estudio de los cromosomas con la finalidad de tener una respuesta efectiva al tratamiento.

Debido a su característica difusa, fijar nuevas intervenciones quirúrgicas representa para el equipo multidisciplinario un reto, pues, hay que valorar las secuelas neurológicas presentes, por ello, se busca mediante la radioterapia ofrecer al paciente condiciones de vida diferente $\mathrm{y}$ aumentar con ello años relativamente efectivos al estar libre de los síntomas, esto permite ofrecer calidad de vida durante la existencia de la enfermedad.

\section{Bibliografía.}

Aguilera, M. (2018). Ciencia y Método. México: Limusa.

Alegría-Loyola, M. (2017). Tumores del Sistema Nervioso Central. Rev Med Inst Mex Seguro Soc, 55(3), 330-40.

García, J. (2017). Astrocitoma Difuso. Salud.

Louis, M. (2016). Tumores Cerebrales. Obtenido de MedlinePlus: https://medlineplus.gov/spanish/braintumors.html

Palavero, C. (2018). Causas de los Astrocitomas. Revista Mexicana de Salud, 36-48.

Tejería, C. (2018). Investigación Científica. Madrid: Pax. 
Génesis Carolina Pérez Cruz; Elizabeth Stefania Molina Vega; Raquel Alexandra Colcha

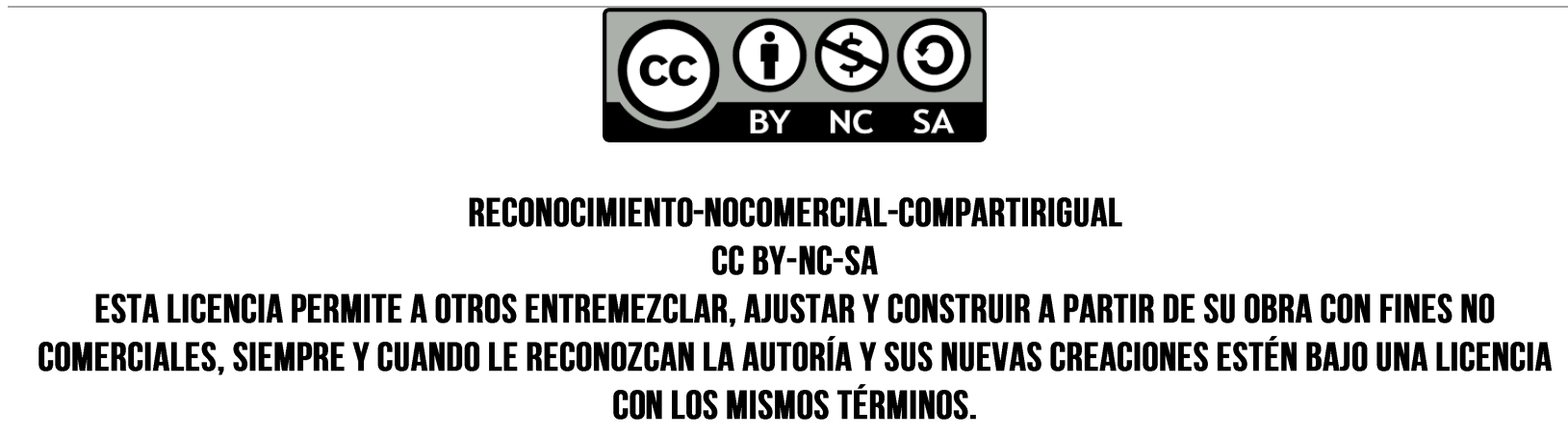

